\title{
Tunable Coplanar Waveguide Microwave Devices on Ferromagnetic Nanowires
}

\author{
Monika Sharma, Bijoy K. Kuanr, Manish Sharma, and Ananjan Basu
}

\begin{abstract}
Ferromagnetic nanowires (FMNW) in porous alumina template are excellent candidates for monolithic integrated microwave devices that can be tuned by material properties and also by externally applied magnetic field. We designed, fabricated and characterized coplanar waveguide (CPW) structures on FMNW substrates. Ni and Co nanowires are grown by electrodeposition method and their surface morphology is determined using scanning electron microscope. We observed that operational frequency of $\mathrm{Ni}$ nanowire based band stop filter increases linearly with applied magnetic field and has very wide frequency tunability. Complex microwave permeability is calculated from the observed transmission response.
\end{abstract}

Index Terms-Ferromagnetic resonance, nanowires, coplanar waveguide, s-parameters.

\section{INTRODUCTION}

Ferromagnetic nanomaterials have been recently studied to large extent as promising candidates for frequency-agile microwave circuit applications such as phase shifters [1], filters [2], isolators [3] and circulators [4]. Magnetic materials have played an important role in addressing problems in electronics technology of the continued increase in the operational frequency in gigahertz range and degree of integration. Band stop filter can reject unwanted carrier frequencies as now a day's microwave devices are widely used in radar systems and in both military and civilian communication systems. The ferromagnetic nanowires embedded in dielectric matrix have interesting properties like high saturation magnetization, high aspect ratio and shows ferromagnetic resonance at high frequencies even without applying external magnetic field which makes them advantageous than ferrites [5], [6]. These substrates can be fabricated at low cost, at room temperature using electrochemical techniques, and they can exhibit high remanence due to their shape anisotropy [7], [8]. Due to easy implementation of additional microwave components without via holes, coplanar waveguide $(\mathrm{CPW})$ structure is especially suitable for the magnetic monolithic integrated circuits (MMIC) design.

In the present work, we have fabricated and characterized microwave band stop filters on porous anodic alumina (AAO) substrate filled with ferromagnetic nanowires (FMNW). Highly ordered nickel and cobalt nanowires are successfully grown in AAO templates by electrochemical

Manuscript received May 5, 2013; revised July 13, 2013.

Monika Sharma, Manish Sharma, and Ananjan Basu are with Centre for Applied Research in Electronics, Indian Institute of Technology Delhi, India (e-mail: monikasharma1604@gmail.com, manish@care.iitd.ac.in, ananjan@care.iitd.ernet.in).

Bijoy K. Kuanr is with University of Delhi, India (e-mail: bkuanr@uccs.edu). deposition. Their surface morphological properties are determined using scanning electron microscope (SEM). The CPW transmission line is patterned on the FMNW substrate and the S-parameters of the device under test are observed by vector network analyzer in frequency range from 10 $\mathrm{MHz}$ to $40 \mathrm{GHz}$. Tunability of the device is measured by applying an external magnetic field. The resonance frequency of ferromagnetic materials is used to calculate the complex permeability of the nanowires.

\section{EXPERIMENT}

\section{A. Ferromagnetic Nanowire Substrate}

A wide range of porosities, heights of membrane, pore sizes and pore space distributions are available [9]-[11]. In this work, anodic alumina membrane from Whatman Ltd. with a nominal pore diameter of $200 \mathrm{~nm}$ and inter pore spacing of $300 \mathrm{~nm}$ is used. A thin layer of $\mathrm{Au}$ film about $150 \mathrm{~nm}$ was sputtered on one side of the template to serve as the working electrode. The electrodeposition of $\mathrm{Ni}$ and $\mathrm{Co}$ nanowire arrays were performed in a three electrode system. The platinum strip was used as counter electrode and saturated calomel electrode (SCE) served as reference electrode. The typical electrolyte solution contains $\mathrm{NiSO}_{4}$ $(330 \mathrm{~g} / \mathrm{L}), \quad \mathrm{NiCl}_{2}(45 \mathrm{~g} / \mathrm{L})$ and $\mathrm{H}_{3} \mathrm{BO}_{3}(38 \mathrm{~g} / \mathrm{L})$ for $\mathrm{Ni}$ nanowires and $\mathrm{COSO}_{4}(120 \mathrm{~g} / \mathrm{L})$ and $\mathrm{H}_{3} \mathrm{BO}_{3}(45 \mathrm{~g} / \mathrm{L})$ for $\mathrm{Co}$ nanowires respectively. The deposition was performed at room temperature and potential was kept at $-0.9 \mathrm{~V}(\mathrm{Ni})$ and $-1.5 \mathrm{~V}$ (Co) versus SCE. The SEM was done using ZEISS EVO 50, the nanowires embedded in AAO was partially dissolved in $3 \mathrm{M} \mathrm{NaOH}$ solution for one hour.

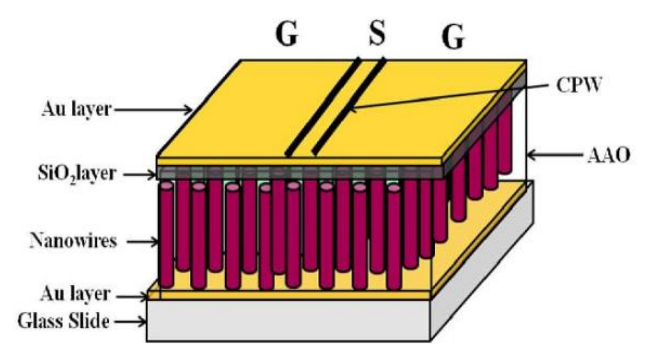

Fig. 1. Schematic representation of band stop filter. GSG is the ground.

\section{B. Band Stop Filter Fabrication}

The CPW lines were fabricated by first depositing a 200 $\mathrm{nm} \mathrm{SiO}_{2}$ layer followed by a metal layer deposition by $\mathrm{rf}$ sputtering. The device was photo-lithographically patterned followed by wet chemical etching technique. The CPW structure was designed for a characteristic impedance of 50 $\Omega$ by adjusting the width of the central signal line and the gap between the signal line and the ground planes. The width of signal line was $155 \mu \mathrm{m}$ and length of conductor 
line was $25 \mathrm{~mm}$ (see Fig. 1). The schematic of the designed device fabricated on FMNW substrate is shown in Fig. 1. We have also fabricated a meander line (bended) CPW structure for observing the effect of external magnetic field on FMNW substrate. The aim of designing the bended CPW structure (Fig. 2) is to increase the length of line thereby increasing the volume of the magnetic substance under it.

The device was characterized using a $40 \mathrm{GHz}$ Agilent vector network analyzer and a microprobe station. Noise, delay due to uncompensated transmission lines connectors, and frequency dependence in the lines has been taken into account by performing short-open-load-thru (SOLT) calibration using Impedance Standard Substrate (ISS). As a consequence of the fabrication process, the FMNW is directly embedded into the substrate. This leads to the concept of integrated magnetic monolithic microwave device. The electromagnetic wave from a network analyzer was made to propagate through the transmission line. The device was subjected to a static magnetic field upto $4.5 \mathrm{kOe}$ by permanent magnets. The reflection $\left(S_{11}\right)$ and transmission $\left(\mathrm{S}_{21}\right)$ coefficients was measured at different fields. The network analyzer sends an oscillating current which creates a sinusoidal magnetic field $h_{r f}$ that generates a resonant motion of magnetization in the nanowires.

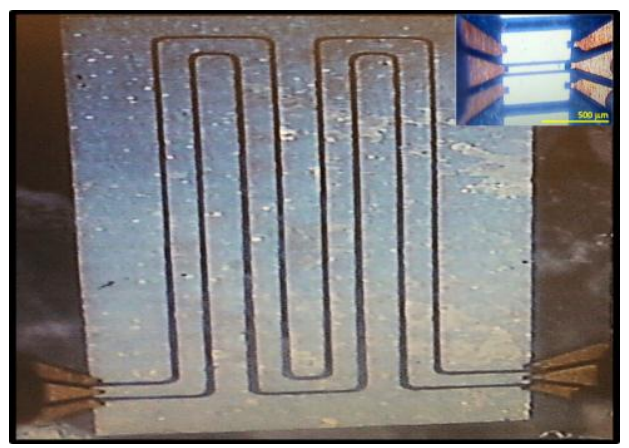

Fig. 2. Microscope image of the device under test with GSG probes for transmitting the response to vector network analyzer and signal planar contacts of coplanar waveguide.

\section{RESULTS AND DisCUSSION}

\section{A. Characterization of Ferromagnetic Nanowires}

Fig. 3(a) shows the SEM image of top view of anodic alumina template; the pores are closely packed with inter-pore spacing of around $300 \mathrm{~nm}$. Fig. 3(b) and (c) reveals top and cross section view of Co nanowires, it is clear that the diameter of the nanowires is about $200 \mathrm{~nm}$. Fig. 3(d) shows highly ordered freely standing Ni nanowires after AAO is partially etched. The length of the nanowires was controlled by deposition time and it is $15 \mu \mathrm{m}$ for Co nanowires and $20 \mu \mathrm{m}$ for $\mathrm{Ni}$ nanowires.

\section{B. Microwave Measurements}

To observe ferromagnetic resonance (FMR), the EM-wave must enter inside the Ni FMNW to be absorbed. A skin depth $(\delta)$ calculation for $\mathrm{Ni}$ (which is frequency dependent $), \delta=(2 / \mu \sigma \omega)^{1 / 2}$ gives $\delta$ about 0.4 microns at the FMR frequency. Since the resulting nanowires have diameter $(200 \mathrm{~nm})$ smaller than skin depth at FMR frequency, the electromagnetic wave can propagate into the substrate. So, FMNW substrate can be used for planar microwave devices.
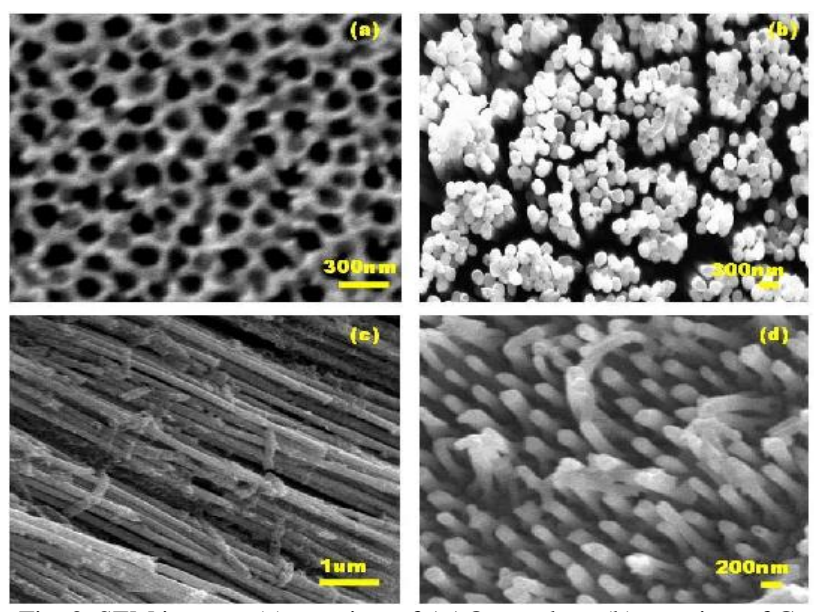

Fig. 3. SEM images: (a) top view of AAO template, (b) top view of Co nanowires embedded in AAO, (c) cross section view of the AAO template filled with Co nanowires, and (d) Ni nanowires partially etched in AAO.
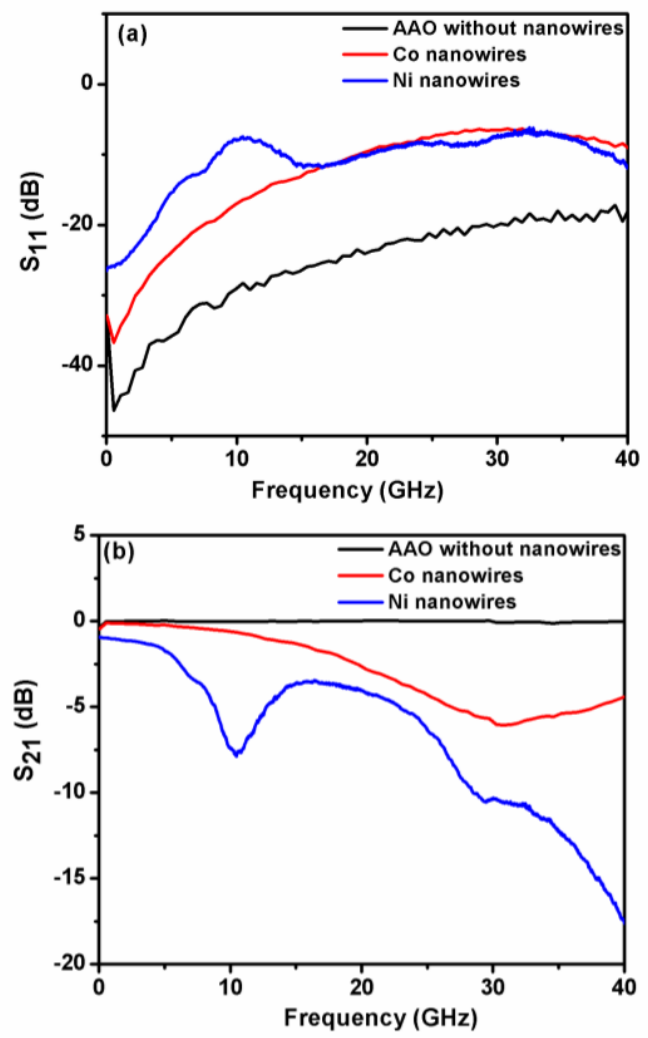

Fig. 4. S-parameters of band stop filter: (a) reflection coefficient, (b) transmission coefficient, for substrates having AAO without nanowires, Co and Ni nanowires.

The S-parameters spectra for empty AAO template and embedded with Ni and Co nanowires (grounded CPW - Fig. 1) are shown in Fig. 4 with zero magnetic field applied. The resonance frequency occurs at $10 \mathrm{GHz}$ for $\mathrm{Ni}$ nanowires and at $30 \mathrm{GHz}$ for Co nanowires. In Fig. 4(a), there is a rise in the reflection $\left(S_{11}\right)$ as the frequency approaches the magnetic material's FMR frequency and the peaks occur about the same frequency as of the FMR frequency. The maximum return loss is about $-7 \mathrm{~dB}$ both for $\mathrm{Ni}$ and $\mathrm{Co}$ NWs based grounded CPW. After the peak, the return loss drops again. The damping in the magnetic material also has an influence on the maximum return loss for the structure. As the damping increases, the maximum reflected signal decreases. This can be seen in a comparison to the bare 
AAO template return loss [black curve - Fig. 4(a)]. The FMR frequency is shown by dip of $S_{21}$ curve [Fig. 4(b)]. The maximum absorption at FMR is about- $8 \mathrm{~dB}$ for Ni NWs and $-6 \mathrm{~dB}$ for Co NWs based filter.

Fig. 5 depicts the absorption characteristics of the meander line CPW. The filter can be tuned with application of magnetic field. The absorption of this filter $(-4$ to -5.5 $\mathrm{dB}$ ) is less than that of the grounded CPW based filter. This may be attributed to the following. The volume of nickel that the EM wave can "sees" determines how much power is lost to the FMNW. As the FMNW are grown from the bottom edge of the CPW (meander line), the EM wave can only "sees" one side of the NWs. The EM wave can penetrates from both sides of the FMNW for the grounded $\mathrm{CPW}$. This may be the reason why we saw more absorption for the grounded CPW based filter.

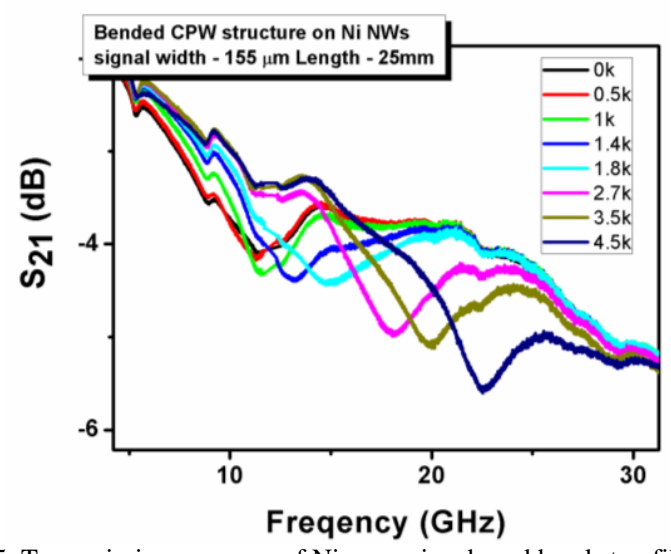

Fig. 5. Transmission response of Ni nanowires based band stop filter at different applied magnetic fields.

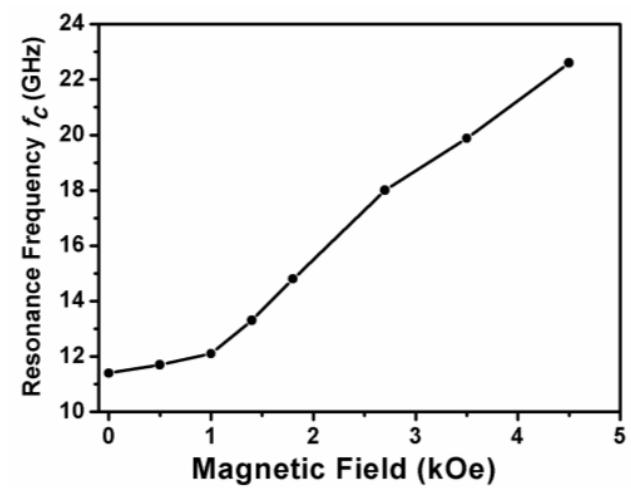

Fig. 6. Ensembles of resonance frequencies as a function of applied magnetic field.

Another important issue is how narrow or wide the transmission dips are? The width of the dip will characterize the rejection band. The rejection band is the range of frequencies that a notch filter effectively blocks out. We measure the rejection band at $-3 \mathrm{~dB}$ of the $S_{21}$ dip. The band width observed is about $5 \mathrm{GHz}$ (Fig. 4).

The key features for the high performance of FMNW based tunable filter are narrow bandwidth for high selectivity, strong FMR absorption for high attenuation, high magnetostriction for wide tunability and high FMR frequency for microwave operation. The selectivity of a filter whether it be band-reject or band-pass is a measure of how narrow the filtering bandwidth is relative to the total operating bandwidth. The higher the selectivity, the higher the filter resolution is. In other words, the better a filter is able to discriminate between one frequency and another. For applications requiring communication channel selection, high selectivity allows for more frequency channels to be packed into the total operating bandwidth. The device operational frequency was also tuned by applying an external magnetic field upto $4.5 \mathrm{kOe}$ for $\mathrm{Ni}$ nanowires as shown in Fig. 5.

Fig. 6 shows the center frequency $\left(f_{c}\right)$ of the filter as a function of the applied field. The resonance frequency showed a linear dependence when the magnetic field is applied parallel to the easy axis of the nanowires. We can conclude from this linearity that the susceptibility and magnetization of the nanowires are small.

The device is characterized as follows. The tunability of a filter is a measure of its ability to shift the center frequency of the filtering bandwidth anywhere within the total operating bandwidth. The general definition for frequency tunability of a filter is -

$$
\frac{f_{c}(\max -\text { field })-f_{c}(\text { zero }- \text { field })}{f_{c}(\text { zero }- \text { field })} \times 100 \%
$$

As the bias field is varied from 0 to $4.5 \mathrm{kOe}$, the center frequency varies from $10 \mathrm{GHz}$ to $22 \mathrm{GHz}$ giving a maximum frequency tunability $>120 \%$. The attenuation of the device enhances by increasing the bias magnetic field by over $40 \%$. The tunable filter requires an external bias to tune the center frequency. To reduce the power consumption of the device it is desirable for the external bias to be as low as possible. At zero magnetic fields the Ni based filter can resonate at $10 \mathrm{GHz}$ and the $\mathrm{Co}$ based at $30 \mathrm{GHz}$. Fig. 7 shows the power losses observed for various filters. Power losses of the device are determined by the relation:

$$
\frac{P_{\text {loss }}}{P_{\text {in }}}=1-\left(\left|S_{21}\right|^{2}+\left|S_{11}\right|^{2}\right)
$$

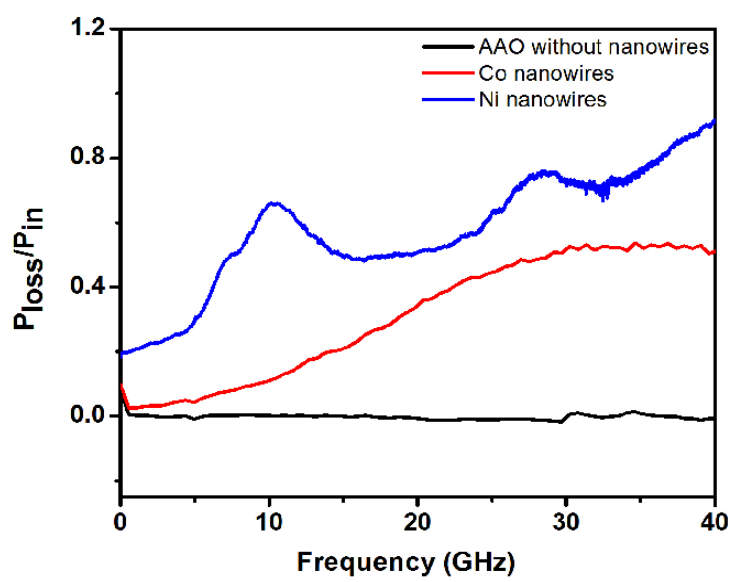

Fig. 7. Power loss of band stop filter: AAO without nanowires, Co and $\mathrm{Ni}$ nanowires.

\section{Microwave Permeability}

The relative magnetic permeability of the ferromagnetic nanowire is a function of frequency $\omega$, the saturation magnetization $\mathrm{M}_{\mathrm{s}}$ and on external applied field $\mathrm{H}_{\mathrm{dc}}$. Relative magnetic permeability is a tensor and assumed to have a form. 


$$
\tilde{\mu}_{f}=\left[\begin{array}{ccc}
\mu_{f} & i k_{f} & 0 \\
-i k_{f} & \mu_{f} & 0 \\
0 & 0 & 1
\end{array}\right]
$$

where $\mu_{f}$ and $k_{f}$ are, respectively, the diagonal and off-diagonal components of the magnetic permeability tensor of one nanowire which are given by relation

$$
\begin{gathered}
\mu_{f}=1+\frac{\omega_{m}\left(\omega_{r}+i \omega \alpha\right)}{\left(\omega_{r}+i \omega \alpha\right)^{2}-\omega^{2}} \\
k_{f}=\frac{\omega_{m} \omega}{\left(\omega_{r}+i \omega \alpha\right)^{2}-\omega^{2}}
\end{gathered}
$$

where $\omega_{m}=\gamma M_{s}$, and $\omega_{r}, \alpha, \gamma, M_{s}$ are the FMR angular resonance frequency, damping factor, gyromagnetic ratio, and the saturation magnetization. The relative permeability of nanowired substrate is evaluated using the following formula:

$$
\mu_{r}=\frac{\mu^{2}-k^{2}}{\mu}, \mu=1+(1-f) \mu_{f}, k=f k_{f}
$$

The filling fraction of nanowires is given by $f$. The real part of relative permeability of nanowire array without applying an external magnetic field is in the range of 0.6-1.6 as shown in Fig. 8. We can easily improve the permeability by applying external field. Therefore FMNW substrates are the best candidate for microwave applications.

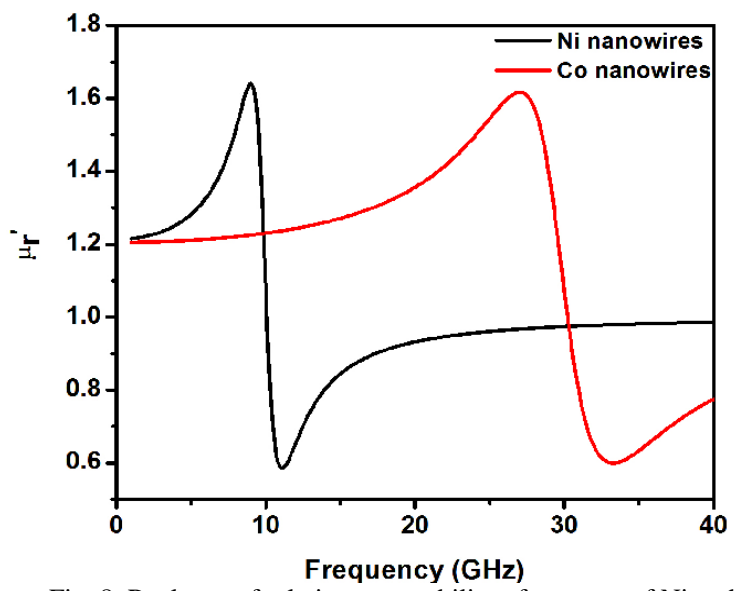

Fig. 8. Real part of relative permeability of an array of Ni and Co nanowires.

\section{CONCLUSION}

In summary, we present a novel fabrication process for ferromagnetic based microwave devices. This process is fully compatible with the present-day IC, PCB and MMIC technology. Band stop filter based on $\mathrm{Co}$ and $\mathrm{Ni}$ nanowires were successfully fabricated. Morphology of the nanowires was characterized by SEM. The working frequency of the device depends upon the material used and can be tuned by applying an external magnetic field. The microwave measurement such as power loss and relative permeability reveals that these substrates are future aspects of microwave devices.

\section{ACKNOWLEDGMENT}

We are thankful for the central facility of SEM of IIT Delhi. We are grateful to the National Programme on Micro and Smart Systems (NPMASS) for setting up RF Characterization facilities and the MEMS Design Centre at the IIT Delhi.

\section{REFERENCES}

[1] Z. H. Wang, Y. Y. Song, Y. Y. Sun, J. S. Bevivino, M. Z. Wu, V. Veerakumar, J. F. Timothy, and E C. Robert, "Millimeter wave phase shifter based on ferromagnetic resonance in a hexagonal barium ferrite thin film," Applied Physics Letters, vol. 97, pp. 72-509, 2010.

[2] B. J. K. Kuanr, V. Veerakumar, K. Lingam, S. R. Mishra, A. V. Kuanr, R. E. Camley, and Z. Celinski, "Microstrip-tunable band-pass filter using ferrite (nanoparticles) coupled lines," IEEE Trans. on Magnetics, vol. 45, pp. 4226, 2009

[3] J. Spiegel, J. D. L. Torre, L. Piraux, and I. Huynen, "Isolator concept based on ferromagnetic nanowired substrates," in Proc. IEEE MTT-S International Microwave Symposium, 2009, pp. 29-32.

[4] A. Saib, M. Darques, L. Piraux, D. V. Janvier, and I. Huynen, "An unbiased integrated microstrip circulator based on magnetic nanowired substrate," IEEE Trans. on Microwave Theory and Techniques, vol. 53, pp. 2043, 2005.

[5] U. Ebels, J. L. Duvail, P. E. Wigen, L. Piraux, L. D. Buda, and K. Ounadjela, "Ferromagnetic resonance studies of Ni nanowire arrays," Physical Review B, vol. 64, pp. 144-421, 2001.

[6] C. A. Ramos, E. V. Brigneti, D. Navas, K. Pirota et al., "Variable-size $\mathrm{Ni}$ magnetic nanowires as observed by magnetization and ferromagnetic resonance," Physica B, vol. 384, pp. 19-21, 2006.

[7] A. Fert and L. Piraux, "Magnetic nanowires," Journal of Magnetism and Magnetic Materials, vol. 200, pp. 338-358, 1999.

[8] H. Chiriac, A. E. Moga, M. Urse, and T. A. Ovari, "Preparation and magnetic properties of electrodeposited magnetic nanowires," Sensors and Actuators A, vol. 106, pp. 348-351, 2003.

[9] X. Wang and G. R. Han, "Fabrication and characterization of anodic aluminum oxide template," Microelectronic Engineering, vol. 66, pp 166-170, 2003.

[10] N. Wang et al., "Fabrication of anodic aluminum oxide templates with small interpore distance," Chinese Physics Letter, vol. 27, pp. 66-801, 2010.

[11] S. H. Su, C. S. Li, F. B. Zhang, and M. S. Yokoyama, "Characterization of anodic aluminum oxide pores fabricated on aluminum templates," Superlattices and Microstructures, vol. 44, pp. 514-519, 2008.

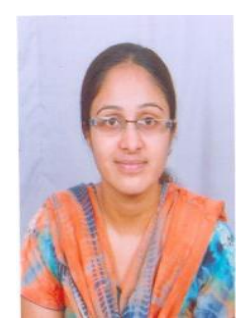

Monika Sharma was born in Delhi, India in 1983. She received the B.Sc. (Hons) degree in Physics and the M.Sc. degree in Physics from University of Delhi, India in 2003 and 2005 respectively. She received her M.Tech. degree in Nanotechnology from National Institute of Technology Kurukshetra, Harayana, India in 2009. She is currently working toward the Ph.D. degree at the Centre for Applied Research in Electronics, Indian Institute of Technology Delhi, India. Her main research concerns modeling, conception, and characterization of devices based on ferromagnetic nanowire array.

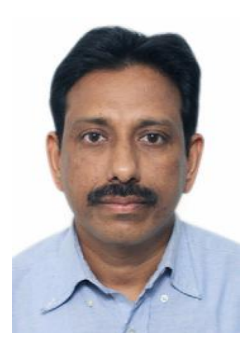

Bijoy K. Kuanr was born in 1962 . He received the Ph.D. degree in Electronic Sciences from the University of Delhi, India in 1993. From 1994 to 1996, he joined the Microwave Laboratory of professor Dr. Güther Nimtz at University of Koeln, Germany as a post-doctoral researcher. From 1999-2001 he worked with Professor Dr. Peter Grünberg-(Nobel Laureate-Physics 2007) as a guest scientist in Ultra-thin Magnetic Multilayer Structures \& GMR-Sensor project. In 2002 he joined University of Colorado at Colorado Springs as a senior research associate. He is also a permanent faculty in the Electronics Department of Zakir Husain Delhi College at Delhi University. His main research deals with electromagnetic theory and measurement techniques applied to materials, devices, and circuits at microwave, millimeter-wave. He has particular interest in the development of microwave 
and millimeter-wave devices based on nanoscaled materials for telecommunication applications. The author is a Member of IEEE.

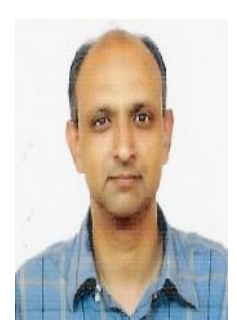

Manish Sharma was born in 1970. He did his B.Tech. (Hons) in Electrical Engg. from IIT Kharagpur in 1991. He worked in the IC industry for three years before joining graduate studies in Stanford University in 1994. There, Manish did an M.S. and Ph. D. in Electrical Engg. 1996 and 2000. During 2000-2006, he was at HP Research Labs in Palo Alto, CA working on materials for magnetic RAM (MRAM), nanolithography and on MEMS-based biological sensors. Since 2006, he has been at IIT Delhi, first with the Dept. of Physics and now at the Centre for Applied Research in Electronics, where he is currently an associate professor.

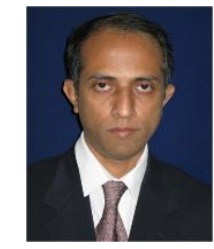

Ananjan Basu was born in Aug. 12, 1969. He did B.Tech. in Electrical Engineering and M.Tech. in Communication and Radar Engineering from I.I.T. Delhi in 1991 and 1993 respectively, and Ph.D. in Electrical Engineering from University of California, Los Angeles in 1998. He has been employed at the

Centre for Applied Research in Electronics, I.I.T. Delhi as an assistant professor from 2000 to 2005 , and as an associate professor from 2005 to 2012 and professor from 2013. His specialization is in Microwave and Millimeter-wave component design and characterization, and antenna development. 